\title{
Proposta de indicadores e padrões para a avaliação de qualidade da atenção hospitalar: o caso da asma brônquica
}

\author{
Indicators and standards proposal for the \\ evaluation of the quality of hospital care: \\ the case for bronchial asthma
}

Marina Ferreira de Noronha 1

Cristiani Vieira Machado 2

Luciana Dias de Lima 3

\footnotetext{
1 Departamento de Administração e Planejamento em Saúde, Escola Nacional de Saúde Pública,

Fundação Oswaldo Cruz. Rua Leopoldo Bulhões 1480, Rio de Janeiro, RJ 21041-210, Brasil.

2 Rua Real Grandeza 281/102, Rio de Janeiro, RJ 22281-031, Brasil.

3 Rua Baronesa de Uruguaiana 44/401, Rio de Janeiro, RJ 20710-310, Brasil.
}

Abstract The objectives of this article are to propose indicators for evaluation of the quality of hospital management of bronchial asthma patients, based on explicit criteria from literature reviews. The central problem identified in the literature review is the erroneous evaluation of severity of asthma crises, either by patients and their rel atives, or by health professionals at all levels of care, causing serious consequences not only for the patient, but for society as a whole. Mortality figures indicate that from 1980 to 1990, an average of 2000 deaths per year from asthma occurred in Brazil, of which 70\% occurred in hospital. Asthma was the fourth cause of hospitalization (hospital admissions), in the state of Rio de Janeiro in 1993. Only $12 \%$ of the admissions that resulted in death made use of the ICU. The above information highlights the need for a thorough evaluation of hospital care of bronchial asthma in Brazil, including a review of all admissions resulting in death and reviews of a sample of all bronchial asthma admissions. Proposed criteria are for this evaluation include: severity of the crise, treatment prescribed, information given to the patient and their relatives, and follow-up appointments made after discharge from hospital.

Key words Bronchial Asthma; Hospital Care Evaluation; Quality Evaluation

Resumo Este artigo tem por objetivos propor indicadores para a avaliação de qualidade da assi stência hospitalar a pacientes com asma brônquica, através de critérios explíci tos baseados em revisão bi bliográfica. O problema central da atenção apontado pela bibliografia foi a existência de fal has na avaliação da gravidade da crise de asma, tanto por parte dos pacientes efamiliares, como pel os profissionais de saúde de todos os níveis da atenção, resultando em sérias conseqüências não só para o paciente, como também para a sociedade em geral. No Brasil, de 1980 a 1990, ocorreram em média 2.000 óbitos por asma/ano, sendo cerca de 70\% deles intra-hospitalares. O diagnóstico de asma foi a quarta causa deinternação no Estado do Rio de Janei ro em 1993. Somente $12 \%$ dos 81 casos que foram a óbi to fizeram uso de UTI. Essas informações justificam uma avaliação mais apurada da assi stência hospitalar a essa doença, e, como contribuição para o processo de avaliação, propomos a realização de revisão da internação de todos os casos que resultaram em óbi to e revisão esporádi ca de uma amostra das internações. Os critérios propostos são: aval iação da gravidade da crise, aval iação da terapia medicamentosa prescrita, educação do paciente e/ou familiares e agendamento de consulta pós-alta hospitalar.

Palavras-chave Asma Brônquica; Avaliação da Atenção Hospitalar; Avaliação deQualidade 
Introdução

A asma brônquica é uma doença pulmonar crônica geralmente não progressiva, caracterizada por crises potencialmente reversíveis de redução do calibre brônquico, devido à hiperreatividade e à inflamação das vias aéreas, como resposta a uma variedade de estímulos físicos, químicos e nervosos. O tratamento atual prescrito para a doença é descrito em diversos protocolos elaborados por sociedades de especialistas. A doença pode ocorrer em todas as faixas etárias, apresentando um espectro de sinais e sintomas cuja gravidade varia de paciente para paciente e também ao longo da vida de cada um. Em circunstâncias raras, episódios agudos podem ser fatais (ATS, 1987; McFadden \& Gilbert, 1992; SBAI/SBP/SBPT, 1994; Campos, 1994; DHHS, 1991; CRPHF, 1993; BTS, 1990; Smith, 1995).

Apesar de a asma raramente evoluir para o óbito, foi observado um aumento da mortalidade pela doença a partir da década de 60 em diversos países, como Reino Unido, Irlanda, Nova Zelândia, Austrália e Noruega (Burr, 1987; Speizer et al., 1968; Speizer, 1987; Beasley et al., 1990; Jackson et al., 1982; Mitchell et al., 1990). Outros países, como os EUA, Canadá e França, não apresentaram essa "epidemia de óbitos" nos anos 60 , mas mostraram elevação na mortalidade por asma nas décadas subseqüentes (Weiss \& Wagener, 1990; Buist, 1990; Sly, 1992; CDC, 1992; Mao et al., 1987; Bousquet et al., 1987).
O aumento da mortalidade por asma suscitou uma profunda discussão acerca dos possíveis fatores envolvidos. Dentre as diversas hipóteses levantadas para explicar esta tendência, podemos citar:

- Alterações na prevalência ou na gravidade da doença.

- Variação dos critérios de codificação ou de diagnóstico.

- Tratamento inadequado e fatores nocivos relacionados à terapêutica.

A partir de revisão bibliográfica, verificouse que a hipótese explicativa mais importante refere-se à possibilidade de fatores relacionados ao tratamento terem levado a uma maior freqüência de óbitos, seja pelo manejo inadequado da doença, seja em conseqüência da adoção de práticas terapêuticas inadequadas (Burr, 1987; Serafini, 1992; Benatar, 1986; McFadden \& Gilbert, 1992; Sears, 1988; Higenbottam \& Hay, 1990). Portanto, mesmo considerando que o aumento da mortalidade por asma em vários países seja multifatorial, torna-se essencial rediscutir o papel da atenção médica e os possíveis "fatores evitáveis" relacionados com os óbitos pela doença.

No Brasil, é marcante a carência de publicações acerca da epidemiologia da asma. Um dos poucos trabalhos foi realizado por Oliveira (1988), que descreveu a evolução da mortalidade por asma da população de cinco a 34 anos do Município e Estado de São Paulo, de 1975 a 1983. O estudo mostrou que as taxas de mortalidade no período mantiveram-se em níveis

Tabela 1

Distribuição dos óbitos por asma por local de ocorrência e por faixa etária, de 1980 a 1990, no Brasil.

\begin{tabular}{|c|c|c|c|c|c|c|c|c|c|c|c|}
\hline Idade & $\begin{array}{c}1980 \\
n\end{array}$ & $\begin{array}{c}1981 \\
n\end{array}$ & $\begin{array}{c}1982 \\
n\end{array}$ & $\begin{array}{c}1983 \\
n\end{array}$ & $\begin{array}{c}1984 \\
n\end{array}$ & $\begin{array}{c}1985 \\
n\end{array}$ & $\begin{array}{c}1986 \\
n\end{array}$ & $\begin{array}{c}1987 \\
n\end{array}$ & $\begin{array}{c}1988 \\
n\end{array}$ & $\begin{array}{c}1989 \\
n\end{array}$ & $\begin{array}{c}1990 \\
n\end{array}$ \\
\hline$<$ lano & $283(52)$ & $257(36)$ & $302(38)$ & $259(43)$ & $249(45)$ & $205(36)$ & $188(33)$ & $165(24)$ & $170(24)$ & $161(20)$ & $179(22)$ \\
\hline $1-4$ & $258(55)$ & $272(54)$ & $289(51)$ & $297(62)$ & $253(53)$ & 261 (49) & $218(41)$ & $215(35)$ & $232(51)$ & $203(41)$ & 212 (43) \\
\hline $5-9$ & 37 (8) & $25(3)$ & $29(7)$ & $30(2)$ & $33(3)$ & $32(7)$ & $29(2)$ & $30(4)$ & $43(5)$ & $26(4)$ & $27(8)$ \\
\hline $10-19$ & $53(17)$ & $46(16)$ & 49 (15) & $51(13)$ & $52(12)$ & 45 (13) & $49(4)$ & $45(9)$ & $28(11)$ & $62(15)$ & 47 (11) \\
\hline $20-39$ & $180(46)$ & $174(52)$ & $175(56)$ & $171(47)$ & $181(50)$ & $160(42)$ & $181(47)$ & $189(45)$ & $157(31)$ & $202(44)$ & $182(44)$ \\
\hline $40-49$ & $152(35)$ & $143(36)$ & $146(31)$ & $163(34)$ & $142(38)$ & $125(35)$ & 145 (39) & $139(29)$ & $139(34)$ & $137(36)$ & 146 (35) \\
\hline $50-64$ & $445(121)$ & $379(100)$ & $412(130)$ & $398(101)$ & 351 (98) & $337(104)$ & $410(111)$ & $304(70)$ & $335(84)$ & $337(81)$ & 367 (117) \\
\hline$>65$ & 814 (327) & 737 (322) & 722 (313) & $813(324)$ & 718 (306) & 725 (314) & 763 (316) & $649(270)$ & 778 (299) & 710 (297) & $833(327)$ \\
\hline Ignorado & $9(2)$ & $14(7)$ & $8(1)$ & $11(3)$ & $11(2)$ & $4(1)$ & $9(3)$ & $11(1)$ & $7(3)$ & $11(4)$ & $8(0)$ \\
\hline Total & $2.286(663)$ & $2.047(626)$ & $2.132(642)$ & $2.193(629)$ & $1.990(607)$ & $1.894(601)$ & $1.992(596)$ & $1.747(487)$ & $1.889(542)$ & $1.949(542)$ & $2.001(607)$ \\
\hline
\end{tabular}

$\mathrm{n}$ = número total de óbitos ocorridos no hospital e fora deste; entre parênteses, somente óbitos ocorridos fora do hospital (no domicílio, via pública, outros e ignorado).

Fonte: Estatística de mortalidade, Ministério da Saúde, AB/Consultoria \& Software. 
"aceitáveis", nunca ultrapassando o coeficiente de 0,9/100.000 habitantes, apesar de apresentarem uma tendência ascendente.

Dados trabal hados pelos autores mostram que, no período de 1980 a 1990, foi registrada uma média de 2.000 óbitos por ano, cuja causa básica foi asma brônquica, e aproximadamente $70 \%$ destes ocorreram dentro de hospitais (Tabela 1). Houve uma redução de $26 \%$ na taxa de mortalidade, passando de 1,9 para 1,4/ 100.000 habitantes entre 1980 e 1990. As regiões brasileiras onde ocorreram as maiores taxas de mortalidade em 1990 foram as Sul e Sudeste (1,8 e $1,5 / 100.000$ habitantes), mas as regiões que apresentaram o maior número absoluto de óbitos foram a Sudeste e Nordeste, com 969 e 492 óbitos respectivamente. De 1980 a 1986, houve um aumento discreto nas taxas de mortalidade por asma na faixa de cinco a 34 anos $(0,19 / 100.000$ habitantes em 1980 e 0,23/ 100.000 habitantes em 1986), estando em níveis abaixo daqueles de diversos países citados neste artigo. Já nas faixas etárias de menores de um ano e maiores de 70, as taxas mantiveram-se elevadas de 1980 a 1990, embora com tendência decrescente.

Comparando com outros países, chama a atenção o excesso de óbitos por asma no Brasil nas faixas etárias mais jovens. A sobremortalidade deste em relação ao Canadá foi de 5, 8 vezes na faixa de zero a 15 anos em 1987. Em relação à França, na faixa de zero a quatro anos, a sobremortalidade no Brasil, masculina e feminina, foi de respectivamente 28,8 e 32,3 vezes no ano de 1984 (Wilkings,1993; Bousquet,1987; Estatística de mortalidade, Ministério da Saúde, estimativa populacional Dr. Kaisô I wakami Beltrão).

Vale ainda ressaltar que a asma foi a quarta principal causa de internação, enquanto diagnóstico principal, nos hospitais conveniados ao SUS do Estado do Rio de Janeiro em 1993, totalizando 24.962 internações com 2,4\% do total de internações (1,9 hospitalizações/ 1.000 habitantes). Os hospitais privados contratados foram responsáveis por $61 \%$ de todas essas internações. Mais da metade das internações (56\%) ocorreram na faixa etária de menores de cinco anos (Tabela 2). Nesse mesmo ano, ocorreram 81 óbitos nos hospitais do SUS, distribuídos em 51 hospitais da rede, perfazendo uma letalidade hospitalar de 0,33\%.

Em 1993, foram pagos US\$4.214.377,45, referentes a internações com o diagnóstico de asma, aos hospitais do Estado do Rio de Janeiro credenciados ao SUS, perfazendo uma média de US\$168,83 por cada internação. Esse valor representou $16,8 \%$ dos gastos incorridos com as internações por doenças respiratórias e 2,3\% dos gastos totais com internações nesse estado (Noronha apud Krauss et al., 1994.

A partir de extensa revisão bibliográfica acerca do processo de tratamento da asma brônquica e do levantamento de dados concernen-

Tabela 2

Distribuição das internações e óbitos por asma*, por faixa etária e natureza jurídica do hospital,

ocorridos em hospitais conveniados ao SUS, no Estado do Rio de J aneiro, 1993.

\begin{tabular}{|c|c|c|c|c|c|c|c|c|c|}
\hline \multirow[t]{2}{*}{ Faixa etária } & \multicolumn{9}{|c|}{ N atureza J urídica } \\
\hline & $\begin{array}{c}\text { Próprio } \\
n\end{array}$ & $\begin{array}{c}\text { Contratado } \\
n\end{array}$ & $\begin{array}{c}\text { Federal } \\
n\end{array}$ & $\begin{array}{c}\text { Estadual } \\
n\end{array}$ & $\begin{array}{c}\text { Municipal } \\
n\end{array}$ & $\begin{array}{c}\text { Filantrópico } \\
n\end{array}$ & $\begin{array}{c}\text { Universit. } \\
n\end{array}$ & $\begin{array}{c}\text { Unid. Pesq. } \\
n\end{array}$ & $\begin{array}{c}\text { Total } \\
\mathrm{n}\end{array}$ \\
\hline$<$ lano & $87(1)$ & $1.181(0)$ & $9(0)$ & $146(0)$ & $304(2)$ & 387 (3) & $24(0)$ & $123(1)$ & $2.261(7)$ \\
\hline $1-4$ & $248(1)$ & $7.871(9)$ & $49(0)$ & $390(0)$ & $1.443(0)$ & $1.089(0)$ & $75(0)$ & $490(2)$ & $11.655(12)$ \\
\hline $5-9$ & $58(0)$ & $1.874(1)$ & $7(0)$ & $99(0)$ & $319(0)$ & $353(0)$ & $32(0)$ & $92(0)$ & $2.834(1)$ \\
\hline $10-19$ & $23(0)$ & $902(0)$ & $9(0)$ & $51(0)$ & $229(0)$ & $231(0)$ & $13(0)$ & $78(0)$ & $1.536(0)$ \\
\hline $20-29$ & $2(0)$ & $790(1)$ & $2(0)$ & $72(0)$ & $271(0)$ & $220(0)$ & $17(0)$ & $74(0)$ & $1.448(1)$ \\
\hline $30-39$ & $6(0)$ & $889(3)$ & $4(0)$ & $110(0)$ & $301(0)$ & $209(0)$ & $18(0)$ & $78(1)$ & $1.615(4)$ \\
\hline $40-49$ & $8(1)$ & $581(8)$ & $2(0)$ & $73(0)$ & $246(1)$ & $193(1)$ & $10(0)$ & $83(0)$ & $1.196(11)$ \\
\hline $50-69$ & $16(0)$ & 858 (15) & $5(0)$ & $114(2)$ & 331 (5) & $312(2)$ & $25(0)$ & $108(1)$ & $1.769(25)$ \\
\hline$>70$ & $25(0)$ & $267(12)$ & $1(0)$ & $28(1)$ & $121(4)$ & $140(3)$ & $18(0)$ & $32(0)$ & $634(20)$ \\
\hline Ignorado & $0(0)$ & $3(0)$ & $0(0)$ & $0(0)$ & $8(0)$ & $3(0)$ & $0(0)$ & $0(0)$ & $14(0)$ \\
\hline Total & $473(3)$ & $15.216(49)$ & $88(0)$ & $1.085(3)$ & $3.573(12)$ & $3.137(9)$ & $232(0)$ & $1.158(5)$ & $24.962(81)$ \\
\hline
\end{tabular}

*Internação em cuja AlH constava o diagnóstico de asma CID 4930, 4931 e 4939.

$\mathrm{n}=$ número total de internações; entre parênteses, número total de óbitos.

AlH normal. Exclui as especialidades: psiquiátrica, crônicos e fora de possibilidade terapêutica.

Fonte: Fita reduzida AIH - DATASUS, AB/Consultoria \& Software. 
tes a esse problema de saúde no Brasil, verificou-se a pertinência em se avaliar a atenção prestada no País a essa doença, tanto em nível ambulatorial como em nível hospitalar. Este trabalho visa contribuir com uma proposta de indicadores e padrões de qualidade, construídos a partir de critérios explícitos, para auxiliar na avaliação do processo de atenção hospitalar a pacientes com asma brônquica. Inicialmente foram apresentadas algumas informações sobre a doença no Brasil e em outros países. Em seguida, fez-se uma revisão da atenção prestada à asma, com algumas informações, também, da atenção no Estado do Rio de Janeiro, finalizando com os indicadores propostos.

\section{Avaliação do tratamento da asma brônquica}

O aumento da mortalidade por asma a partir da década de 60 observado em diversos países impulsionou a discussão acerca de quais fatores relacionados à atenção médica poderiam ter contribuído para a maior freqüência de óbitos pela doença. É de certa forma consensual que os pacientes com crise grave que são prontamente atendidos em unidades hospitalares especializadas raramente morrem se submetidos à avaliação e tratamentos apropriados (Serafini, 1992). Nesse contexto, foram realizados estudos de avaliação da atenção aos casos que evoluíram para o óbito ocorrido dentro ou fora do hospital, assim como dos casos que foram internados em hospitais e que não tiveram esse desenlace, visando identificar possíveis faIhas na atenção (fatores evitáveis).

Um ponto a ser considerado na análise desses estudos é o caráter crônico e flutuante da asma, o que torna difícil precisar qual parcela da mortalidade pela doença pode ser atribuída às falhas relacionadas ao manejo crônico ou à conduta frente à crise aguda.

Sabe-se que parte significativa dos óbitos conseqüentes à crise asmática aguda ocorre sem que o paciente tenha recebido atendimento médico hospitalar. Em estudos realizados em regiões da Inglaterra com dados do final das décadas de 60 e de 70, o percentual de óbitos ocorridos fora do hospital variou entre $73 \%$ na faixa etária de cinco a 34 anos e de $86 \%$ na faixa de 15 a 64 anos (Fraser et al., 1971; Ormerod \& Stableforth, 1980; BTA, 1982) e foi de $85 \%$ na Nova Zelândia (Sears, 1988). Já no Estado de Oregon, nos EUA, o percentual de óbitos ocorridos fora do hospital foi de $31 \%$ no ano de 1982 (Barger et al., 1988). Dados brasileiros sobre a mortalidade no período de 1980 a 1990 demonstram que cerca de $30 \%$ dos óbitos por asma ocorrem fora do hospital (variando de
487 a 663 óbitos/ano) (Tabela 1). Analisando separadamente os Estados do Rio de Janeiro e São Paulo, em ambos, esse percentual foi cerca de $25 \%$ no ano de 1990, destacando a importância da avaliação de qualidade da assistência, uma vez que no Brasil a maior parte dos pacientes que evoluem para o óbito chegam a receber algum atendimento médico hospitalar.

Segundo a literatura, em algumas situações a evolução da crise aguda é muito rápida e fulminante, impedindo que medidas sejam tomadas em tempo hábil. Esses casos, entretanto, correspondem a uma minoria. Muitos dos pacientes com essa evolução, apresentavam história prévia de episódio de gravidade semeIhante ao que levou ao óbito e a ocorrência de sinais de alarme precedendo ao óbito, como instabilidade marcante com variação diurna ou piora gradativa da obstrução brônquica (BTA, 1982; Stableforth, 1983; Hetzel et al., 1977; Westerman et al., 1979), o que deveria implicar um controle mais rigoroso da doença no nível ambulatorial e domiciliar. Mesmo assim, Johnson et al. (1984), avaliando noventa óbitos por asma ocorridos na faixa etária de 15 a 64 anos, nas regiões inglesas de West Midlands e Mersey, no ano de 1979, consideraram dez óbitos como provavelmente inevitáveis. Em cinco desses casos, o paciente morava só efoi encontrado já morto; nos demais, o óbito ocorreu no espaço de trinta minutos do início da crise. MacDonald et al. (1976), em Cardiff (Inglaterra), e pesquisadores de países como os EUA e Canadá também encontraram um percentual de óbitos com evolução muito rápida da crise fatal (Barger et al., 1988; Collins-Williams et al., 1981).

\section{Avaliação do tratamento ambulatorial e domiciliar}

A asma, enquanto doença crônica, pressupõe acompanhamento a longo prazo e implica necessariamente anotações e realização de exames no decorrer da enfermidade, para que se possa avaliar corretamente a gravidade desta, permitindo um planejamento adequado tanto do tratamento medicamentoso, como da educação do paciente e/ ou familiares para o seu manejo a longo prazo (Speizer et al., 1968). Sheffer \& Taggart (1993) relatam que os cuidados da asma pressupõem um manejo gradativo, durante o qual o número de medicações e a freqüência de administração são aumentados quando a gravidade da doença aumenta ou vice-versa, até que o controle da asma seja mantido. As medidas de função respiratória são parâmetros de avaliação objetivos, importantes 
para se definir quais os passos a seguir no tratamento da doença. Todos os pacientes com asma que requerem terapia diária, devem ser monitorados utilizando parâmetros objetivos, como o uso de medidor de fluxo expiratório.

A avaliação inadequada, tanto por parte dos médicos, como pelos pacientes, tem resultado em complacência e atraso no reconhecimento de uma crise grave e na implementação de terapia apropriada.

O estudo de MacDonald et al. (1976) ilustra bem as fal has mais comuns verificadas no atendimento ambulatorial, e também aquelas ocorridas no atendimento hospitalar. Esses autores, utilizando informações de 1963 a 1974, verificaram que, de 83 pacientes que foram a óbito, $60 \%$ tiveram história de internação hospitalar prévia, internações essas muitas vezes recentes, sugerindo a instalação de doença grave. $\mathrm{Na}$ ocasião do ataque fatal, $47 \%$ dos pacientes estavam em uso de corticóide por via oral, embora freqüentemente em doses inferiores às preconizadas para a asma classificada como grave, e $70 \%$ em uso de broncodilatador. Os autores sugerem que os pacientes que já haviam sido previamente internados não receberam orientação adequada e medicação suficiente para o controle de sua doença, apontando para falhas na avaliação da gravidade da doença. Esse mesmo estudo também mostra falhas na avaliação da gravidade no momento da crise fatal quando, dos 21 pacientes que foram avaliados por médicos, 43\% (nove) não foram encaminhados para internação hospitalar. Também foram verificadas falhas na avaliação da gravidade do episódio fatal pelo doente e/ ou familiares, resultando em demora na solicitação de atendimento médico. Outros estudos realizados no Reino Unido com informações da década de 70 encontraram resultados similares aos do estudo de M acDonald et al. (BTA, 1982; Johnson et al., 1984; Ormerod \& Stableforth, 1980).

Fraser et al . (1971), avaliando óbitos ocorridos na Inglaterra em 1968-1969, encontraram evidências de que a inalação excessiva de broncodilatadores pode ter contribuído para a ocorrência de óbitos, o mesmo encontrando Collins-Williams et al. (1981) no Canadá. Esses autores concluem que a razão principal para o excesso de uso de broncodilatadores parece estar relacionada à falha do paciente em apreciar que, quando tal medicamento não é eficaz em reverter os sintomas, é sinal de necessidade de introdução de outro tratamento e não de aumento da dose, apontando, portanto, para faIhas na orientação de pacientes e/ ou familiares.
Barger et al. (1988), analisando óbitos ocorridos em 1982 nos EUA, verificaram que, no acompanhamento da doença, $78 \%$ dos pacientes estavam fazendo uso de teofilina e somente $50 \%$ haviam realizado dosagem de teofilina sérica em algum momento do manejo crônico. Somente $41 \%$ dos pacientes haviam realizado estudos de função pulmonar ao longo do tratamento. Verificaram também que pacientes mais idosos tiveram um tratamento medicamentoso menos agressivo tanto no manejo crônico, como na crise fatal.

Os dados acima enfatizam que os problemas referentes ao manejo crônico da asma e à abordagem da crise aguda estão intimamente relacionados. Falhas no manejo ambulatorial da asma podem repercutir negativamente nos resultados da atenção hospitalar, já que pacientes mal controlados tendem a ser considerados como de maior risco. Além disso, o manejo crônico inadequado da asma pode acarretar um aumento no número de internações.

\section{Avaliação das internações por asma}

Cochrane \& Clark (1975), avaliando o tratamento hospitalar dispensado a 19 pacientes na faixa de 35 a 64 anos que foram a óbito no ano de 1971 em hospitais da Grande Londres, concluíram que, embora os óbitos em sua maior parte tenham sido considerados repentinos e inesperados, não se poderia excluir possíveis fal has na avaliação da gravidade da crise. Isso poderia ter levado a falhas no tratamento efetivo dos pacientes. Os autores verificaram que, no momento da admissão, somente dois pacientes tiveram anotadas informações suficientes para se avaliar a gravidade e somente $26 \%$ (cinco) tiveram medidas de função pulmonar anotadas, havendo dois outros casos no decorrer da internação. Ainda na admissão, foram realizados quatro exames de gasometria e, posteriormente, mais dois, tendo sido feito Raio X de tórax na maioria dos pacientes (17). No momento do óbito, havia uma subprescrição de medicamentos, sendo que $42 \%$ dos pacientes não estavam fazendo uso de corticóide, e somente $52 \%$ estavam usando broncodilatador oral e/ ou na forma inalante. Quinze pacientes fizeram uso de sedativos, embora somente um caso tenha si do encaminhado a CTI. O uso de sedativos foi avaliado como causa de depressão respiratória em dois casos e razão do início da deterioração das condições clínicas em quatro casos. Os autores levantam a hipótese de que as falhas na avaliação dos pacientes com asma, possam dever-se à baixa letalidade hospitalar e à experiência comum de 
se assistir à maior parte dos casos se recuperarem de uma crise grave, levando os profissionais a subestimarem a possibilidade de morte por asma. Sugerem que, na Inglaterra, os óbitos por asma sejam tratados com a mesma atenção e publicidade dispensada aos óbitos maternos e também que sejam transformadas em rotina as avaliações de pico de fluxo expiratório e gasométricas.

Ormerod \& Stableforth (1980), avaliando 53 óbitos por asma em Birmingham (Reino Unido) no período de 1975 a 1977, detectaram fatores evitáveis em 15 casos ocorridos dentro do hospital, os principais fatores foram: avaliação inicial inadequada, considerando-se a subutilização de espirometria e gasometria (50\%), subutilização de corticóides (93\%) e de broncodilatadores por via intravenosa e respiratória (100\%) e falhas na monitorização objetiva da eficácia do tratamento (100\%).

Eason \& Markowe (1987) realizaram estudo de caso-controle avaliando 35 óbitos hospitalares na faixa de cinco-45 anos, ocorridos na região de North East Thames (Inglaterra), durante um ano, entre 1982 e 1983. Consideraram $46 \%$ dos óbitos potencialmente evitáveis, encontrando fal has no manejo da crise de asma em $83 \%$ dos casos. As principais falhas segundo a equipe revisora foram: a) investigação inicial da crise inadequada, uma vez que $71 \%$ dos casos que foram a óbito não tiveram avaliação da função pulmonar (34\% dos controles) e $66 \%$ não realizaram exame de gasometria (34\% dos controles); b) inadequado monitoramento da função pulmonar ao longo da internação (71\% dos casos e $23 \%$ dos controles) e da gasometria (63\% dos casos e $89 \%$ dos controles); c) inadequado tratamento com broncodilatadores, já que $49 \%$ dos casos não receberam tratamento com broncodilatador inalatório, contra $11 \%$ dos controles; d) inadequado tratamento com corticosteróide, pois $28 \%$ dos casos ( $17 \%$ dos controles) não receberam essa medicação nas vias oral ou parenteral, embora houvesse indicação; e) dosagem excessiva de aminofilina ( $40 \%$ dos casos e $20 \%$ dos controles); f) nãoinstituição de ventilação mecânica em nove casos, o que pode ter contribuído para o óbito.

Spevetz et al. (1992), avaliando 130 internações de pacientes em Status Asthmaticus, de 1989 a 1990, em um hospital universitário de New Jersey (EUA), verificaram que apenas $68 \%$ dos casos tiveram registro de avaliação do pico de fluxo expiratório em algum momento da internação. Nenhum paciente teve registro de espirometria no primeiro dia de internação, 22\% o tiveram em dias subseqüentes e somente um paciente teve estudo espirométrico seqüencial.
Foram anotadas informações sobre sibilos em $100 \%$ das internações, mas informações muito mais importantes para uma avaliação adequada da gravidade tiveram sub-registro, como o uso ou não de musculatura acessória registrado em somente $65 \%$ dos casos (65\%), presença ou não de pulso paradoxal (26\%) e diaforese (19\%).

Daley et al. (1991), na avaliação retrospectiva da atenção hospitalar a 127 pacientes com o diagnóstico de asma aguda, em três hospitais universitários de Boston (EUA), observaram diferenças significativas na abordagem diagnóstica e na freqüência de utilização de exames. Os autores enfatizaram que a espirometria, embora sendo o teste mais diretamente relacionado à gravidade da crise asmática, não foi utilizada rotineiramente pelos hospitais. A freqüência de sua utilização nas primeiras 12 horas de admissão foi de: $47 \%$ × $23 \% \times 26 \%$ dos casos em cada hospital. A medida do volume expiratório realizada na emergência ocorreu em $29 \%$, $23 \%$ e $23 \%$ dos casos respectivamente.

Oliveira (1988), em avaliação retrospectiva de prontuários, descreve o tratamento de 12 casos que foram a óbito em um hospital do Estado de São Paulo entre 1970 e 1973. Verificou que mais da metade dos casos (sete) faleceram nas primeiras 24 horas de internação. Quanto à avaliação da gravidade da crise, somente dois casos tiveram avaliação da função pulmonar anotada, não foi feita qualquer anotação sobre pulso paradoxal, uma anotação sobre uso de musculatura acessória, e em oito casos anotou-se a alteração de consciência. Em seis pacientes realizou-se gasometria; em quatro, Raio $X$ de tórax. Todos os casos receberam broncodilatador, mas somente na forma inalatória; nove receberam corticosteróide e seis receberam sedativo. Quatro pacientes fizeram uso de ventilação mecânica. Pode-se verificar que as falhas no tratamento hospitalar são semelhantes às encontradas nos estudos já descritos acima.

A internação hospitalar é considerada um momento propício à educação dos pacientes, à reavaliação da medicação utilizada e ao planejamento da conduta a longo prazo. Bucknall et al. (1988), através de entrevistas realizadas com pacientes asmáticos duas semanas após a alta de um hospital da Escócia, observaram falhas significativas na educação dos pacientes, acerca da compreensão e gerência da asma. Verificaram que pouquíssimos pacientes possuíam o medidor de fluxo expiratório para uso em sua moradia, embora 32\% tivessem história de ataques repentinos e crises graves. Somente 13\% dos pacientes relataram terem sido instruídos 
a iniciar com corticóide oral na ocorrência de um novo ataque grave. Verificaram também que $34 \%$ dos entrevistados não tinham um plano apropriado no caso de uma crise. A maioria dos pacientes não tinha idéia de como os seus medicamentos agiam e poderiam auxiliá-los.

Atenção à asma no Estado do Rio de J aneiro

Foram utilizadas informações contidas nos formulários de Autorização de Internação Hospitalar - AlH - do SIH-SUS, apresentados no ano de 1993, referentes a internações ocorridas em hospitais conveniados ao SUS no Estado do Rio de Janeiro. O banco de dados foi composto das AlHs reduzidas (não contêm todas as informações), tendo sido selecionados os dados das AlHs-1 (normais ou de identificação) e utilizado o programa computacional SGT (Sistema Gerador de Tabelas) da empresa A/B Consultoria e Software, para manuseio desses dados.

Foram analisadas todas as internações que tinham anotadas como diagnóstico principal um dos três códigos da CID- 9 referentes à asma brônquica (CID 493.0, 493.1 e 493.9) e as análises referem-se aos três códigos agrupados. Neste artigo, serão apresentadas somente algumas informações sobre a atenção prestada aos casos que evoluíram para o óbito.

Ocorreram 81 óbitos com o diagnóstico de asma brônquica. Para saber se os pacientes que foram a óbito chegaram ao hospital já em estado grave, foi verificado inicialmente o tempo médio que estes permaneceram hospitalizados até a ocorrência do óbito. Observou-se que o tempo médio de permanência - TMP geral foi de 3,5 dias, sendo que, na faixa etária de 40-49 anos, o TMP foi de 5,7 dias, ao passo que, na de menores de um ano, foi de 5,3 dias; já na faixa de um a quatro anos, na qual ocorreram 12 óbitos, o TMP foi de 1,2 dia, sugerindo uma maior gravidade na admissão hospitalar.

Verificou-se que, dos 81 óbitos, 33 aconteceram dentro das primeiras 48 horas de internação; destes, 16 foram referentes a pacientes que deram entrada no hospital em estado agônico e 17 em estado não agônico. A maior parte dos óbitos (48) ocorreu acima de 48 horas da internação. Portanto, pode-se supor que somente $20 \%$ dos casos que foram a óbito deram entrada no hospital em estado grave, embora não seja possível afirmar que o restante também não tenha sido internado em estado grave, pois faltam informações mais precisas do que se tenha considerado como agônico. Enfim, embora com limites, essas informações mostram que $80 \%$ dos pacientes que foram a óbito tiveram seu estado agravado dentro do hospital, abrindo a hipótese da possibilidade de ocorrência de um desenlace diferente.

Somente $12 \%$ (dez) dos pacientes que foram a óbito utilizaram a unidade de terapia intensiva - UTI . Dos dez casos que fizeram uso de UTI, seis pertenciam à faixa etária de menores de cinco anos. Vale destacar que nenhum dos 16 pacientes que chegaram agônicos ao hospital e faleceram dentro das primeiras 48 horas recebeu atendimento em unidade de terapia intensiva; dos 17 que não internaram em estado agônico mas faleceram nas primeiras 48 horas, somente dois utilizaram UTI. Considerou-se esta taxa baixa, na medida em que se espera que esses pacientes que foram a óbito tenham tido necessidade de utilização de tecnologias mais complexas como a oferecida em unidades de terapia intensiva. Sugere-se que esta baixa taxa de utilização seja, em parte, explicada pela inexistência desse cuidado especializado na grande maioria dos hospitais financiados pelo SUS no Estado do Rio de Janeiro, embora este devesse ser um dos requisitos para o credenciamento de hospitais ao atendimento de pacientes com asma em estado grave.

As informações apresentadas acima sugerem que pode estar havendo problemas de qualidade na atenção hospitalar a pacientes com asma brônquica no Estado do Rio de Janeiro, necessitando, portanto, que seja feita uma averiguação mais detalhada dessa atenção.

Além desses resultados, os vários estudos citados nos tópicos anteriores, apontaram para a ocorrência de falhas em todo o espectro da atenção ao paciente asmático, tanto em décadas distantes, como em mais recentes. Foi verificado que diversos óbitos poderiam ter sido evitados, tendo sido identificados alguns fatores da atenção que contribuíram para tal. Foram verificadas fal has dos profissionais, tanto no atendimento ambulatorial, como no hospitalar e nas avaliações da gravidade tanto da doença, quanto da crise. Essas falhas repercutiram, principalmente, na utilização de instrumentos para avaliação da gravidade da obstrução brônquica, na prescrição medicamentosa, no encaminhamento dos pacientes e na educação de pacientes e/ ou familiares. Foram também verificadas falhas dos pacientes e/ ou familiares na avaliação da crise de asma. 
Indicadores para monitorar a qualidade da atenção hospitalar aos pacientes com asma

Avaliar a qualidade implica mensurar um evento e emitir um juízo acerca deste. Para tal, há necessidade de se estabelecer critérios relevantes em termos de qualidade desse evento e padrões indicando os limites aceitáveis e inaceitáveis de qualidade. Os critérios são caracterizados como explícitos e implícitos. O primeiro tipo implica o estabelecimento, anteriormente à avaliação, de regras claras dos componentes a serem avaliados e os limites de aceitabilidade; quanto ao segundo tipo, fica a cargo do avaliador, geralmente um especialista, o estabel ecimento dos critérios (Donabedian, 1980; Vuori, 1989).

A partir da revisão bibliográfica e de análise de informações sobre a mortalidade por asma no Brasil e da atenção ao paciente asmático nos hospitais do SUS no Rio de Janeiro, descritas anteriormente, propõem-se al guns indicadores para monitorar a qualidade da atenção hospitalar aos pacientes com asma. Decidiu-se por sugerir critérios genéricos para a avaliação do processo de atenção à asma, priorizando aspectos críticos do tratamento, identificados pelos estudos consultados, sem descer a detalhes considerados mais adequados de ser abordados, seja por especialistas na área, utilizando critérios implícitos, seja tendo como referência os protocolos de atenção.

Selecionaram-se assi $m$ indicadores que podem ser utilizados tanto por profissionais que estão envolvidos com o atendimento a pacientes asmáticos, como pelas chefias de unidades de atenção a doenças respiratórias ou pelas comissões de avaliação da qualidade da atenção hospitalar. Alguns desses indicadores são úteis não apenas ao gerente hospitalar, mas também aos gerentes dos níveis regional e central, que dispõem de Sistemas de Informações para a aplicação de alguns desses indicadores, como o Sistema de Informação de Mortalidade (SIM) e das Internações Hospitalares (AlHs).

Optou-se por sugerir duas abordagens para a avaliação da qualidade da atenção hospitalar a esses pacientes:

I. Revisão dos casos de óbito na perspectiva de eventos-sentinela.

II. Revisão esporádica das internações.
I. Primeira abordagem: óbitos por asma como eventos-sentinela

Eventos-sentinela são aqueles desnecessários e indesejáveis do ponto de vista da saúde, cuja ocorrência representa um alerta da provável existência de problemas na qualidade da assistência, justificando a sua avaliação para a identificação de fatores que devam ser corrigidos. Rutstein et al. (1976) propuseram a utilização de mortes evitáveis enquanto eventos-sentinela em meados da década de 70 , sendo seguidos por outros autores (Holland, 1988; Westerling \& Smedby, 1992; Westerling, 1992).

Segundo Rutstein et al. (1976), considerar um óbito como evitável significa assumir que ele poderia ter sido prevenido se tudo tivesse transcorrido adequadamente, havendo responsabilidade, ao menos parcial, do serviço de saúde sobre esse desdobramento. Esses autores não apenas selecionaram algumas doenças cujas mortes seriam possivel mente evitáveis, como procuraram apontar em que circunstâncias estas seriam julgadas preveníveis. Já naquela época, a morte por asma foi considerada como evitável em pessoas abaixo de cinqüenta anos que evoluíram para o óbito após automedicação de broncodilatador por via inalatória. É importante destacar que, na ocasião, havia grande preocupação com o uso excessivo de broncodilatadores, o que poderia ter sido parcialmente responsável pelo aumento da mortalidade por asma registrado na década precedente em diversos países, como Reino Unido, Nova Zelândia, etc.

Apesar de Rutstein et al. (1976) terem sido restritivos na aplicação do conceito de morte evitável para a asma, os diversos estudos já descritos acerca das circunstâncias de ocorrência dos óbitos em asmáticos sugerem a ampliação das condições a serem monitoradas. Nesse contexto, optou-se por utilizar a concepção de morte evitável num sentido mais abrangente, assumindo que todos os óbitos por asma sejam potencialmente preveníveis e sugerindo a revisão minuciosa de todos os casos de óbito por asma em busca de quaisquer falhas da atenção, passíveis de correção. Procurou-se então enumerar alguns critérios explícitos para essa revisão, conforme o quadro a seguir. Cabe ressaltar que a aplicação desses critérios deve ser feita por especialistas experientes, os quais estariam habilitados para avaliar as peculiaridades de cada caso. Deve-se também observar que os dois primei ros critérios são de exclusão, isto é, excluem a possibilidade de se evitar a ocorrência do óbito. 
Critérios propostos para revisão

dos casos de óbito

1) Intervalo de tempo entre o início da crise e a ocorrência do óbito.

2) Gravidade da crise no momento da internação.

3) Avaliação da gravidade da crise:

Critérios clínicos: uso de musculatura acessória, presença de pulso paradoxal, diaforese. Critérios objetivos: avaliação espirométrica e/ou medição do pico defluxo expiratório.

4) Avaliação da terapia medicamentosa prescrita: uso de broncodilatadores $\beta_{2}$-agonistas, uso de corticosteróide.

Intervalo de tempo entre o início da crise e a ocorrência do óbito

Quando se analisam as causas que levaram pessoas ao óbito por alguma doença, há necessidade de se estabelecerem critérios para avaliar se esses seriam ou não inevitáveis. Segundo a literatura já apresentada em item anterior deste artigo, em algumas situações, a evolução da crise aguda de asma é fulminante, impedindo que medidas sejam tomadas em tempo hábil.

Johnson et al. (1984) apontaram como óbitos inevitáveis aqueles ocorridos no intervalo menor ou igual a trinta minutos a partir do início dos sintomas, assumindo que pouco poderia se fazer nos casos de ataques fulminantes, mesmo que estes tenham conseguido chegar ao hospital. Vale ressaltar que esses casos de evolução fulminante podem estar relacionados a falhas anteriores no acompanhamento, sendo comum entre esses pacientes a história de internações prévias pela doença. Entretanto, para efeito da avaliação da internação em questão, optou-se por assumir como óbitos inevitáveis aqueles decorrentes de ataques fulminantes conforme definido por Johnson et al. (1984).

Gravidade da crise no momento da internação

Pacientes que, independentemente da duração da crise, chegam ao hospital agônicos (com insuficiência respiratória, distúrbios da consciência ou com doenças graves associadas ao estado de descompensação) e evoluem para o óbito em menos de trinta minutos da entrada no hospital, mesmo tendo recebido assistência imediata e adequada, podem ser considerados como mortes inevitáveis na perspectiva da avaliação desta internação.
Avaliação da gravidade da crise

Depois de confirmado o diagnóstico de asma brônquica, a avaliação da gravidade da crise vem sendo considerada como um dos aspectos mais importantes para a escolha da conduta terapêutica e controle da resposta ao tratamento. A avaliação inadequada da gravidade e, conseqüentemente, a não-instituição do tratamento adequado podem gerar transtornos à vida do paciente, aos familiares, à sociedade em geral, gastos financei ros desnecessários, sobrecarga nas instituições de prestação de serviços de saúde e resultar em óbitos potencialmente evitáveis.

Um instrumento que possivelmente auxilia no tratamento e acompanhamento do paciente, além de permitir fazer comparações, é a utilização de uma forma padronizada de avaliação da intensidade da crise de asma. Há na bibliografia diversas formas de se classificar a gravidade tanto da doença,como da crise de asma brônquica. Essas classificações variam de acordo com os critérios utilizados, tendo como referência o grau de dependência do paciente para desenvolver as suas atividades diárias (Jones, 1971 apud Cochrane \& Clark, 1975); os resultados de exames espirométricos (Westerman et al., 1979); o histórico de utilização de medicamentos e da morbidade (Janson-Bjerklie et al., 1992) e classificações que mesclam critérios objetivos e clínicos, considerando o desenvolvimento anterior da doença, o seu estado atual e fatores de risco de morte, apresentadas pelos diversos protocolos de atenção à asma brônquica (DHHS, 1991; SBAI/SBP/SBPT, 1994; Campos, 1994; CRPHF, 1993; BTS, 1990). Vale ressaltar que muitas das classificações correntes na bibliografia necessitam de validação.

Neste ponto é fundamental que se focalize a atenção ao tratamento da crise de asma grave, já que é esta a que necessita de internação mais freqüentemente e, portanto, a escolhida como objeto desse estudo. Alguns critérios são considerados fundamentais para definição dos pacientes com crise de asma grave. Esses crité rios devem ser utilizados durante o atendimento de emergência para que possam ser adotadas as medidas e condutas pertinentes ao caso. O DHHS (1991), no “Protocolo para o diagnóstico e gerência da asma”, aponta para diversos fatores que favorecem a hospitalização.

Todos os protocolos utilizam, no geral, critérios semelhantes para caracterização de uma crise grave de asma. Vale ressaltar o da British Thoracic Society (BTS, 1990), que dividiu os sinais de crise de asma grave em sinais de morte potencial e sinais de morte iminente. 
A anormalidade funcional na asma é a obstrução geralmente reversível das vias aéreas; portanto, os sinais e sintomas decorrentes do desenvolvimento de obstrução das vias aéreas necessitam ser identificados e esta deve ser tratada adequadamente, antes que evolua e complique a ponto de colocar em risco a vida do paciente. Este é um indicador fundamental para se avaliar a gravidade de uma crise asmática, ressaltando que mesmo pacientes assintomáticos podem ter obstrução significativa.

Um dos sinais de obstrução das vias aéreas é a redução do volume expiratório forçado no primeiro minuto $\left(V_{E} F_{1}\right)$ e a redução do pico de fluxo expiratório (PFE), que podem ser verificados através de medidas de espirometria ou através de medidor de pico de fluxo expiratório que avalia o PFE, sendo estas duas avaliações referidas como "objetivas" pelos diversos autores. O aparelho de medida do fluxo expiratório foi desenvolvido entre as décadas de 40 e 50. Em fins da década de 50, Wright \& McKerrow (1959) apresentaram uma forma portátil para utilização hospitalar e ambulatorial. Em 1978, Wright apresentou o minimedidor de pico de fluxo expiratório possibilitando o seu uso individual na moradia do paciente (Wright, 1978 apud Eichenhorn et al., 1982). O medidor de pico de fluxo expiratório é volume e esforço dependentes, mas, apesar dessas limitações, é uma medida útil do índice de obstrução das vias aéreas, correlacionando razoavelmente bem com o VEF 1 (Van As, 1982). Eichenhorn et al. (1982) encontraram variação na acurácia e confiabilidade entre três modelos de medidores de PFE. Mesmo assim, um desses aparelhos cumpriu todos os critérios estabelecidos pela British Thoracic Society. Ao longo desses anos, tem havido uma atenção maior no aprimoramento desses aparelhos.

Uma grande discussão na bibliografia é a capacidade dos médicos de avaliarem clinicamente o grau de obstrução das vias aéreas, como dito anteriormente, aspecto importante da avaliação da gravidade da crise asmática. Um dos sinais clínicos de obstrução brônquica é a presença de sibilos, embora a sua ausência não signifique que não esteja havendo obstrução. Shim \&Williams (1983) avaliaram a relação entre os sibilos e a gravidade da obstrução brônquica. Verificaram que a presença de sibilos expiratórios e inspiratórios está associada a um valor mais reduzido do pico de fluxo expiratório do que a presença somente de sibilos expiratórios.

Neste mesmo estudo, os autores buscaram verificar a variação da observação da intensidade dos sibilos entre uma equipe de médicos especialistas experientes, pois, para que a avaliação clínica dos sibilos tenha utilidade na caracterização da obstrução brônquica, o resultado dessa avaliação teria que ser homogêneo entre os clínicos. Ao testar a variação da observação clínica, verificaram grande variabilidade entre os observadores, comprometendo portanto, a sua utilidade. Os autores concluíram que, na avaliação do paciente, há necessidade de utilização de métodos objetivos (PFE e / ou $V F_{1}$ ) de mensuração da obstrução brônquica.

Esses mesmos autores desenharam outro estudo buscando testar a acurácia de clínicos e pacientes em estimar os valores de pico de fluxo expiratório. Selecionaram para tal exercício especialistas experientes em doenças respiratórias e pacientes portando asma crônica. Somente $44 \%$ das estimativas do valor de PFE realizadas pelos especial istas chegaram a um resultado próximo de $20 \%$ do valor obtido pela mensuração do PFE. Quanto à avaliação dos pacientes do seu próprio valor de PFE, foi encontrada uma correlação positiva entre a percepção destes e o valor obtido pela mensuração do PFE. Os autores concluíram que pacientes experientes foram muito mais acurados em avaliar o seu valor de PFE do que clínicos especialistas, mostrando-se acurados também em julgar se a obstrução estava mel hor, pior ou sem alteração no seu dia-a-dia (Shim \& Williams, 1980).

O estudo supracitado utilizou pacientes que já tinham prática no uso do medidor de pico de fluxo expiratório e provavelmente estes pacientes foram bem instruídos ao longo de sua doença, aprendendo a reconhecer melhor seus sintomas. Os resultados de estudos das circunstâncias dos óbitos citados anteriormente neste artigo verificaram um resultado diferente quanto à avaliação de pacientes da gravidade de sua crise, mostrando falhas nessa avaliação que, juntamente com outros fatores, podem ter contribuído para o seu óbito. Esses mesmos estudos confirmaram a dificuldade de clínicos em avaliar a gravidade do paciente.

Um dos estudos mais importantes acerca da importância de parâmetros objetivos para a avaliação da gravidade foi o de McFadden et al. (1973). Os autores, comparando o grau de obstrução das vias respiratórias com as manifestações clínicas em 22 pacientes em crise aguda de asma, verificaram que, quando estes ficaram assintomáticos, a função mecânica pulmonar variou de $40 \%$ a $50 \%$ dos valores normais esperados para tais pacientes, demonstrando assim uma obstrução persistente, embora assintomática, das vias respiratórias. Os autores concluem que a ausência de dispnéia e sibilos não garantem a normalidade e que, se a 
suspensão do tratamento for baseada somente nesses sinais, pode-se subestimar seriamente uma grande extensão de doença residual. Neste sentido, torna-se essencial a utilização não só de critérios clínicos, como de parâmetros objetivos na avaliação inicial e seqüencial do paciente internado. Rubinfeld \& Pain (1976) e Murray et al. (1977) reforçam essas conclusões com seus estudos, e Morris et al. (1971) desenvolvem padrões espirométricos.

\section{- Critérios clínicos}

Os critérios para a avaliação da gravidade propostos abaixo são mais indicados para pacientes na faixa etária acima de cinco anos.

Os principais sinais de gravidade da crise comumente citados na literatura são: pulso paradoxal, uso de musculatura acessória e diaforese. A presença ou ausência destes sinais deve estar registrada em todos os prontuários, pelo menos no momento da admissão hospitalar.

Os sibilos constituem o achado mais freqüente nas crises asmáticas, devendo seu registro ser sistemático, tanto no momento da admissão, como ao longo da internação. Entretanto, a presença deste sinal isoladamente não constitui um indicador que seja suficiente enquanto referencial de gravidade. Literatura utilizada para os indicadores acima: Spevetz et al., 1992; ATS, 1987; McFadden \& Gilbert, 1992; SBAI / SBP/ SBPT, 1994; Campos, 1994; DHHS, 1991; CRPHF, 1993; BTS, 1990.

\section{- Critérios objetivos}

Conforme enfatizado acima, a avaliação da gravidade baseada somente em sinais clínicos é insuficiente para a verificação do grau de obstrução brônquica, havendo necessidade da utilização de métodos objetivos, como a medida de pico de fluxo expiratório e/ ou avaliação espirométrica. Ao menos um desses dois exames deve ser realizado e registrado em prontuário no momento da admissão, durante a internação para a monitorização da eficácia terapêutica e antes da alta para garantir a adequação da medicação para uso domiciliar. Como padrão mínimo, espera-se que $100 \%$ dos pacientes acima de cinco anos de idade e que não apresentem problemas físicos que impeçam a realização do exame sejam submetidos a pelo menos uma avaliação objetiva durante a internação, sendo a espirometria o exame de preferência pela sua maior precisão. Esse indicador é importante visto que diversos autores enfatizaram a sua subutilização (Daley et al., 1991; Spevetz et al., 1992; BTA, 1982; Johnson et al., 1984; Bucknall et al., 1988; Lazarus, 1989; Oliveira, 1988).
A gasometria arterial pode fornecer informações adicionais, sendo essencial nos casos de insuficiência respiratória ou evolução desfavorável do quadro asmático. A tele-radiografia de tórax, apesar de estar indicada para o diagnóstico diferencial da asma e detecção de complicações respiratórias, não constitui um exame de grande utilidade para a avaliação da gravidade da crise asmática em si. Este fato merece ser destacado, já que a radiografia é muitas vezes solicitada com maior freqüência que a espirometria, o pico de fluxo expiratório e a gasometria, exames estes mais específicos para a avaliação do quadro asmático (Daley et al., 1991). Outras referências utilizadas foram ATS, 1987; McFadden \& Gilbert, 1992; SBAl/SBP/SBPT, 1994; Campos, 1994; DHHS, 1991; CRPHF, 1993 e BTS, 1990.

Embora não tendo relação direta com a avaliação da gravidade, outro indicador importante da qualidade da atenção e adequado uso de tecnologias pode ser a freqüência da utilização dos exames complementares discutidos acima. Sugere-se que os serviços verifiquem e comparem a freqüência de utilização desses exames.

Avaliação da terapia medicamentosa prescrita

A partir da avaliação da gravidade da crise asmática é traçada a conduta medicamentosa e decidida a necessidade ou não de internação hospitalar.

Uma apreciação da fisiopatologia da asma proporciona a base para a conduta terapêutica. O marco fisiopatológico de uma crise de asma constitui-se de redução do calibre das vias aéreas devido à contração da musculatura lisa, desenvolvimento de edema da árvore brônquica e formação de secreções espessadas, provocando hiperirritabilidade inespecífica da árvore traqueobrônquica, com inflamação das vias aéreas. $\mathrm{O}$ aumento da resistência das vias aéreas, por sua vez, leva a diminuição do fluxo e volume expiratório forçados, hiperinsuflação pulmonar, alterações funcionais da musculatura respiratória, alterações da elasticidade pulmonar, com desequilíbrio da relação ventilação-perfusão e alterações nos gases arteriais $\left(\mathrm{pO}_{2}\right.$ e $\left.\mathrm{pCO}_{2}\right)$. Evidências sugerem que essa hiper-reatividade inespecífica está relacionada a inflamação das vias aéreas.

Alguns aspectos do tratamento da asma foram modificados a partir dessas descobertas sobre sua fisiopatogenia. Por um longo tempo pensou-se que os episódios de crise de asma fossem unicamente decorrentes da obstrução 
respiratória causada pelo broncoespasmo. Baseada neste conceito, a terapia consistia primariamente da utilização de broncodilatadores, tais como os agentes beta agonistas e a teofilina, direcionados para o relaxamento da musculatura lisa das vias aéreas. Mais recentemente, foi reconhecido que a asma brônquica não está relacionada somente ao broncoespasmo. É necessária a existência de um processo inflamatório subjacente nas vias aéreas para a constituição de um quadro de hiper-sensibilidade e hiper-reatividade brônquica. A implicação farmacológica dessa nova abordagem fisiopatológica da doença é que atualmente o tratamento de grande número de pacientes asmáticos inclui agentes antiinflamatórios que reduzem e previnem a recorrência da inflamação e desta forma a broncoconstricção. Dados clínicos sugerem que terapia vigorosa antiinflamatória melhora não somente a função pulmonar, como também pode reduzir o grau de hiperresponsividade da musculatura das vias respiratórias (McFadden, 1991; M cFadden \& Gilbert, 1992; Sheffer \& Taggart, 1993).

Conforme citado na literatura, a base da terapêutica ao paciente em estado de crise asmática que necessite de internação é principalmente constituída de Broncodilatadores $\mathrm{B}_{2^{-}}$ agonistas e de Glucocorti cói des. A título de avaliação de uma internação, a prescrição desses medicamentos pode ser utilizada como parâmetro mínimo de referência da qualidade da atenção, embora muitas vezes haja também indicação de outros medicamentos adicionais. $\mathrm{N}$ ão estamos considerando aqui três fatores de extrema importância que vão depender das características do paciente e do grau de gravidade em que se encontra, quais sejam: dosagem desses medicamentos, vias e freqüência de administração. Pode-se utilizar como referência da dosagem, via e freqüência de administração o ditado pelos diversos protocolos disponíveis (SBAI/SBP/SBPT, 1994; DHHS, 1991; CRPHF, 1993; BTS, 1990; ATS, 1987; Smith, 1995). Praticamente todos os estudos de revisão dos casos de óbito citados anteriormente neste artigo chamam a atenção para a subutilização de corticosteróides durante o período de internação.

II. Segunda abordagem: revisão esporádica das internações

Devido ao fato de os óbitos por asma serem raros, torna-se insuficiente a avaliação somente através destes eventos, havendo necessidade de uma revisão esporádica das internações para complementar o monitoramento da quali- dade da assistência. Para a revisão de prontuários de pacientes que não evoluíram para o óbito, os indicadores propostos são alguns dos já discutidos acima, mantendo-se os mesmos parâmetros, além de dois novos indicadores que são discutidos a seguir.

Critérios propostos para revisão esporádica das internações

1) Avaliação da gravidade da crise: Critérios clínicos: uso de musculatura acessória,presença de pulso paradoxal, diaforese. Critérios objetivos: avaliação espirométrica e/ou medição do pico de fluxo expiratório.

2) Avaliação da terapia medicamentosa prescrita: uso de broncodilatadores $\mathrm{B}_{2}$-agonistas, uso de corticosteróide.

3) Educação do paciente e/ ou familiares: revisão ou elaboração de um plano terapêutico.

4) Agendamento de consulta pós-alta hospitalar.

Os itens 1 e 2 seguem os critérios descritos na primeira abordagem.

\section{Educação do paciente}

A necessidade de educação do paciente asmático vem sendo bastante enfatizada pela literatura, principalmente em artigos de revisões de óbitos (Collins-Williams et al., 1981), nos protocolos de atenção à asma já citados, nos de avaliação da assistência hospitalar (Fitzgerald $\&$ Hargreave, 1989; Bucknall et al., 1988), e em artigos de revisão do tratamento realizados por especialistas (Higenbottam \& Hay, 1990; Campos, 1994; Serafini, 1992). Por ser a asma uma doença crônica, o seu adequado controle pressupõe uma boa compreensão da doença e de seu manejo pelo paciente e/ ou familiares, pois muitos dos desfechos trágicos têm ocorrido devido à desinformação. Todos os protocolos de atenção a pacientes asmáticos consideram como parte integrante do tratamento a educação dos pacientes e/ ou familiares acerca da doença e a elaboração de um plano terapêutico individualizado, sob a forma escrita, voltado para os momentos de crise e tratamento a longo prazo, para auxiliar na gerência da doença. Esse plano se faz imprescindível principalmente para aqueles pacientes identificados como tendo risco de desenvolver uma crise grave de asma.

O período de internação do paciente asmático é uma oportunidade de se avaliar todo o seu processo de tratamento e redirecioná-lo, caso seja necessário, visando à adoção de condutas que levem à estabilização do processo 
asmático e prevenção de futuras crises que possam resultar em novas internações. Constitui um critério de boa qualidade da atenção hospitalar todos os pacientes, no momento da alta, terem em mãos um plano terapêutico indi vidual construído ou revisado durante a internação, contendo principalmente informação acerca de como e onde obter atenção médica urgente e também informações claras sobre o monitoramento dos sintomas, uso do medidor de fluxo expiratório (caso seja factível) e uso de medicamentos. Vale ressaltar que os protocolos e especialistas recomendam que principalmente pacientes com asma crônica grave e/ ou com pouca percepção da gravidade de sua crise devam ter um medidor de fluxo expiratório próprio para uso em sua moradia e fazer uso de um diário de sintomas, devendo ser instruídos para tal.

Agendamento de consulta

O tempo de internação de um paciente com crise grave muitas vezes não é suficiente para que ocorra a total reversão do quadro de obstrução brônquica, havendo necessidade de continuação do tratamento medicamentoso após a alta hospitalar. Portanto, após a internação, há necessidade de monitoração até a cura completa, para que não reste resíduo de obstrução brônquica que, com o tempo, possa tornar-se irreversível (Barnes, 1989; Brown et al., 1984). Torna-se, portanto, recomendável que o paciente tenha alta do hospital com uma consulta agendada dentro da semana seguinte à alta hospitalar (BTS, 1990; Bucknall et al., 1988).

\section{Considerações finais}

Por meio da revisão bibliográfica, pode-se observar que, a partir do aumento do número de óbitos em alguns países da Europa e Oceania na década de 60, iniciou-se um despertar gradual para esse problema de saúde, outrora adormecido no dia-a-dia dos serviços. No Brasil, só recentemente foi divulgado um protocolo de atenção ao paciente com asma, promovido por sociedades de especialistas, e aguardase a divulgação das diretrizes para a educação do paciente, discutidas em encontro recente. Poucos são os trabalhos publicados na área que permitem ter uma visão da dimensão desse problema no País.

A asma brônquica, enquanto uma doença crônica, constitui-se num problema típico de saúde pública nos moldes da diabetes ou da hipertensão arterial. Embora esse problema de- vesse ser gerenciado primordialmente em nível ambulatorial, causa perplexidade que, no Estado do Rio de Janeiro, esta tenha sido a quarta causa de internação, tanto em 1993, quanto em 1994. Na falta de informações mais precisas sobre a confiabilidade desse diagnóstico no banco de dados e de informações sobre a gravidade desses episódios de internação que permitam justificá-las, resta-nos questionar se não estão ocorrendo internações evitáveis. Por outro lado, o grande número de óbitos ocorrendo no País anualmente, em sua maioria dentro de hospitais, justificam uma revisão da atenção a essa doença não só nos hospitais, mas em toda a rede de atenção à saúde.

Ao utilizar-se o banco de dados das AlHs, verificou-se que algumas variáveis podem oferecer informações sobre a qualidade do processo da atenção, como: o cruzamento das variáveis óbito, onde está especificado se este ocorreu dentro de 48 horas ou após esse tempo; se os pacientes estavam agônicos ou não nas primeiras 48 horas, com a utilização de UTI. Pode-se checar a data de admissão com o tempo de permanência em UTI para se ter uma noção do momento de encaminhamento a esse serviço. Tanto nos casos que foram a óbito, quanto nos que não, pode-se verificar a utilização de exames especiais, como: espirometria, gasometria, utilização de ventilação mecânica e outros; pode-se também checar o tempo de permanência hospitalar com o número e tipo de exames e tipo de alta. Há possibilidade de se adicionar a informação de idade e sexo aos cruzamentos acima, identificar os hospitais e verificar alguns de seus recursos de estrutura. Por meio do cruzamento entre o diagnóstico e o procedimento realizado, pode-se ter uma noção de possíveis problemas no diagnóstico de asma ou mesmo possibilidade de fraude, necessitando, entretanto, de uma averiguação em prontuários nos casos em que as duas variáveis não tenham nenhuma compatibilidade. No momento de realização desta pesquisa, os dados das AlHs dispunham de um número reduzido de informações, não sendo possível, por isso, apresentar todos os cruzamentos acima, além de não ser este o objeto principal desteartigo.

O banco de mortalidade foi menos explorado, mas permitiu verificar a proporção de óbitos ocorridos dentro dos hospitais e sua distribuição no País. Como os dados de mortalidade para o ano de 1993 ainda não estavam disponíveis, não se pôde checar o número de óbitos apresentados nas AlHs do Estado do Rio de Janeiro com os contidos para este Estado na estatística de mortalidade. 
Buscou-se identificar indicadores que abarcassem todo o espectro da atenção hospitalar a esse problema de saúde. São indicadores gerais que ainda não foram validados, mas podem auxiliar numa seleção inicial de casos a serem mais profundamente investigados. Para a avaliação dos casos selecionados é necessário desenvolver critérios mais específicos e adequados às realidades dos serviços ou utilizar os protocolos de atenção já validado.

\section{Referências}

ATS (American Thoracic Society), 1987. Standards for the diagnosis and care of patients with chronic obstructive pulmonary disease (COPD) and asthma. American Review Respiratory Disease, 136:225-244.

BALDWIN, D. R.; ORMEROD, L. P.; MACKAY, A. D. \& STABLEFORTH, D. E., 1990. Changes in hospital management of acute severe asthma by thoracic and general physicians in Birmingham and Manchester during 1978 and 1985. Thorax, 45:130134.

BARNES, P. J., 1989. A new approach to the treatment of asthma. New England Journal of Medicine, 321:1.517-1.527.

BARGER, L. W.; VOLLMER, W. M.; FELT, R. W. \& BUIST, S. A., 1988. Further investigation into the recent increase in asthma death rates: a review of 41 asthma deaths in Oregon in 1982. Annals of Allergy, 60:31-39.

BEASLEY, R.; SMITH, K.; PEARCE, N.; CRANE, J.; BURGESS, C. \& CULLING, C., 1990. Trends in asthma mortality in New Zealand, 1908-1986. Medical Journal of Australia, 152:570-573.

\section{Agradecimentos}

Os autores agradecem a Vera Lucia Edais Pepe, Miguel Murat V., Milena Piraccini Duchiade, Mauricio Andrade Pires, Claudia Travassos Veras, Leticia Krauss Silva, Hisbelo Campos S. e Fábio Kushinir, pelas valiosas contribuições.
BENATAR, S. R., 1986. Fatal asthma. New England Journal of Medicine, 314:423-429

BOUSQUET, J.; HATTON, F.; GODARD, P. \& MICHEL, F. B., 1987. Asthma mortality in France. Journal of Allergy and Clinical Immunology, 80:389-394.

BTA (British Thoracic Association), 1982. Death from asthma in two regions of England. British Medical Journal, 285:1.251-1.255.

BTS (British Thoracic Society), 1990. Guidelines for management of asthma in adults: II - acute severe asthma. British Medical Journal, 301:797800.

BROWN, P. J.; GREVILLE, H. W. \& FINUCANE, K. E., 1984. Asthma and irreversible airflow obstruction. Thorax, 39:131-136.

BUCKNALL, C. E.; ROBERTSON, C.; MORAN, F. \& STEVENSON R. D., 1988. Management of asthma in hospital: a prospective audit. British Medical Journal, 296:1.637-1.639.

BUIST, S. A., 1990. Reflections on the rise in asthma morbidity and mortality. Editorial. Journal of theAmerican Medical Association, 264:1.719-1.720. 
BURR, M. L., 1987. Is asthma increasing? Journal of Epidemiology and Community Health, 41:185189.

CAMPOS, H. S., 1994. Asma para clínicos. Jornal Brasileiro de Medicina. 66:161-174.

CDC (Center of Disease Control), 1992. Asthma United States, 1980-1990. MMWR, 41:733-735.

CRPHF (Centro de Referência Professor Hélio Fraga), 1993. Proposta para Padronização do Tratamento Ambulatorial do Asmático. Rio de Janeiro. (mimeo.)

COCHRANE, G. M. \& CLARK, T. J. H., 1975. A survey of asthma mortality in patients between ages 35 and 64 in the greater London hospitals. Thorax, 30:300-305.

COLLINS-WILLIAMS, C.; ZALESKY, C.; BATTU, K. \& CHAMBERS, M. T., 1981. Death from asthma. Canadian Medical Association Journal, 125:341345.

DALEY, J.; KOPELMAN, R. I.; COMEAU, E.; GINNS, L. C. \& ROSSING, T. H., 1991. Practice patterns in the treatment of acutely ill hospitalized asthmatic patients at three teaching hospitals - variability in resource utilization. Chest, 100:51-56.

DHHS (Department of Health and Human Services), 1991. Guidelines for the Diagnosis and Management of Asthma. National Asthma Education Program. Expert Panel Report. Bethesda: DDHS

DONABEDIAN, A., 1980. The Definition of Qualility and Approaches to its Assessment. Ann Arbor: Health Administration Press.

EASON, J. \& MARKOWE, H. L. J., 1987. Controlled investigation of deaths from asthma in hospitals in the North East Thames region. British Medical Journal, 294:1255-1258.

EICHENHORN, M. S.; BEAUCHAMP, R. K.; HARPER, P. A. \& WARD, J. C., 1982. An assessment of three portable peak flow meters. Chest, 82:306-309.

FITZGERALD, M. J. \& HARGREAVE, F. E., 1989. The assessment and management of acute life-threatening asthma. Chest, 95:888-894.

FRASER, P. M.; SPEIZER, F. E.; WATERS, S. D. M.; DOLL, R. \& MANN, N. M., 1971. The circumstances preceding death from asthma in young people in 1968 to 1969. British Journal Disease Chest, 65:71-84.

HETZEL, M. R.; CLARK, T. J. H. \& BRANTHWAITE, M. A., 1977. Asthma: analysis of sudden deaths and ventilatory arrests in hospital. British Medical Journal, 1:808-811.

HIGENBOTTAM, T. \& HAY, I., 1990. Has the treatment of asthma improved? Chest, 98:706-712.

HOLLAND, W. W., 1988. European Community Atlas of Avoi dable Death. Commission of the European Communities Health Services Research, series n.3, Oxford: Oxford University Press.

JACKSON, R. T.; BEAGLEHOLE, R. T.; REA, H. H. \& SUTHERLAND, D. C., 1982. Mortality from asthma: a new epidemic in New Zealand. British Medical Journal, 285:771-774.

JANSON-BJERKLIE, S.; FERKETICH, S.; BENNER, P. \& BECKER, G., 1992. Clinical markers of asthma severity and risk: Importance of subjective as well as objective factors. Heart and Lung, 21:265-272.

JOHNSON, A. J.; NUNN, A. J.; SOMNER, A. R.; STABLEFORTH, D. E. \& STEWART, C. J., 1984. Circumstances of death from asthma. British Med- ical Journal, 288:1870-1872.

LAZARUS, S. C., 1989. Rational therapy of acute asthma. Annals of Allergy, 63:585-590.

MacDONALD, J. B.; SEATON, A. \&WILLIAMS, D. A., 1976. Asthma deaths in Cardiff 1963-74: 90 deaths outside hospital. British Medical Journal, 6.024:1.493-1.495.

MCFADDEN, J. R., 1991. Asthma. In: Principles of Internal Medicine (E. R. Harrison's, ed.), 12a ed., pp. 1.047-1.053, New York: Mcgraw-Hill Book Company.

MCFADDEN, E. R.; KISER, R. \& DEGROOT, W. J., 1973. Acute bronchial asthma. Relations between clinical and physiologic manifestations. New England Journal of Medicine, 288:221-225

MCFADDEN, E. R.; GILBERT, I. A., 1992. Asthma. New England Journal of Medicine, 327:1.928-1.937.

MAO, Y.; SEMENCIW, R.; MORRISON, H.; MacWILLIAM, L.; DAVIES, J. \& WIGLE, D., 1987. Increased rates of illness and death from asthma in Canada. Canadian Medical Association Journal, 137:620-624.

MITCHELL, E. A.; ANDERSON, H. R.; FREELING, P. \& WHITE, P. T., 1990. Why are hospital admission and mortality rates for childhood asthma higher in New Zealand than in the United Kingdom? Thorax, 45:176-182.

MORRIS, J. F.; KOSKI, A. \& JOHNSON, L. C., 1971. Spirometric standards for healthy nonsmoking adults. American Review of Respiratory Disease, 103:57-67.

MURRAY, A. B.; HARDWICK, D. F.; PIRIE, G. E. \& FRASER, B. M., 1977. Assessing severity of asthma with Wright peak-flow meter. The Lancet, 8.013:708.

NORONHA, M. F., 1994. Proposta preliminar de indicadores para a avaliação da atenção hospitalar à asma brônquica. In: Desenvolvimento de um Sistema de Informações Gerenciais para Unidades Hospitalares (L. Krauss; C. Travassos Veras \& M. F. Noronha, orgs.), Rio de Janeiro: Inamps/ Fiocruz. Convênio n.28-92. Relatório Técnico.

OLIVEIRA, M. V. C., 1988. Mortalidade por asma. Revista Paulista de Medicina, 106:247-250.

ORMEROD, L. P. \& STABLEFORTH, D. E., 1980. Asthma mortality in Birmingham 1975-7: 53 deaths. British Medical Journal, 280:687-690.

RUBINFELD, A. R. \& PAIN, M. C. F., 1976. Perception of asthma. The Lancet, 7.965:882-884.

RUTSTEIN, D.; BERENBERG, W.; CHALMERS, T. C.; CHILD, C. G.; FISHMAN, A. P. \& PERRIN, E. B., 1976. Measuring the quality of medical care. A clinical method. New England Journal of Medicine, 294:582-588.

SBAI/SBP/SBPT (Sociedade Brasileira de Alergia e Imunopatologia/Sociedade Brasileira de Pediatria/Sociedade Brasileira de Pneumologia e Tisiologia), 1994. I Consenso Brasileiro no Manejo da Asma. Fortaleza: SBAI/SBP/SBPT.

SEARS, M. R., 1988. Increasing asthma mortality Fact or artifact? Journal of Allergy and Clinical Immunology, 82:957-960.

SERAFINI, U., 1992. Can fatal asthma be prevented? A personal view. Clinical and Experimental Allergy, 22:576-588.

SHEFFER, A. L. \& TAGGART, V. S., 1993. The National Asthma Education Program - expert panel report guidelines for the diagnosis and management of asthma. Medical Care, 31(suppl.):MS20-MS28. 
SHIM, C. S. \&WILLIAMSJR, M. H., 1983. Relationship of wheezing to the severity of obstruction in asthma. Archives of Internal Medicine, 143:890-892.

SHIM, C. S. \& WILLIAMS JR, M. H., 1980. Evaluation of the severity of asthma: patients versus physicians. American Journal of Medicine, 68:11-13.

SLY, R. M., 1992. Asthma mortality, East and West. Annals of Allergy, 69:81-84.

SMITH, S. J., 1995. Asthma management and other highlights of the annual meeting of the American Academy of Allergy and Immunology. Technology News, 8:1-5.

SPEIZER, F. E., 1987. Historical perspectives: the epidemic of asthma deaths in the United Kingdom in the 1960s. Journal of Allergy and Clinical Immunology, 80(part II):368-372.

SPEIZER, F. E.; DOLL, R. \& HEAF, P., 1968. Observations on recent increase in mortality from asthma. British Medical Journal, 5.588:335-339.

SPEVETZ, A.; BARTTER, T.; DUBOIS, J. \& PRATTER, M. $R ., 1992$. Inpatient management of status asthmaticus. Chest, 102:1.392-1.396.

STABLEFORTH, D., 1983. Death from asthma. Editorial. Thorax, 38:801-805.

VAN AS, A., 1982. The accuracy of peak expiratory flow meters. Chest, 82:263.
VUORI, H. V., 1989. El Control de Calidad en Ios Servicios Sanitarios - Conceptos y Metodologia. Editora Masson S.A.

WEISS, K. B. \& WAGENER, D. K., 1990. Changing patterns of asthma mortality. Identifying target populations at high risk. Journal of theAmerican Medical Association, 264:1683-87.

WESTERMAN, D. E.; BENATAR, S. R.; POTGIETER, P. D. \& FERGUSON, A. D., 1979. Identification of the high-risk asthmatic patient. American Journal of Medicine, 66:565-572.

WESTERLING, R., 1992. Trends in "avoidable" mortality in Sweden, 1974-1985. Journal of Epidemiology and Community Health, 46:489-493.

WESTERLING, R. \& SMEDBY, B., 1992. The European Community "Avoidable Death Indicators" in Sweden 1974-1985. International Journal of Epidemiology, 21:502-510.

WILKINGS, K. \& MAO, Y., 1993. Trends in rates of admission to hospital death from asthma among children and young adults in Canada during the 1980s. Canadian Medical Association Journal, 148:185-190

WRIGHT, B. M. \& McKERROW, C. B., 1959. Maximum forced expiratory flow rate as a measure of ventilatory capacity. British Medical Journal, 5.158:1.0411.047 . 